\title{
Clinical Observation of Combined Application of Oxiracetam and Hyperbaric Oxygen Clinical Curative Effect on Vascular Dementia Patients
}

\author{
Guiwen Bu* \\ Department of Internal Medicine, The Hospital of Liuyang County, Changsha, Hunan 410000, China
}

\begin{abstract}
Objective: To evaluate the application of oxiracetam combined with hyperbaric oxygen on vascular dementia clinical curative effect. Method: Three groups of patients were given active treatment basic diseases, including antiplatelet, promote cerebral circulation and cerebral protective agent. And respectively take the hyperbaric oxygen therapy, olathe temple therapy and combination therapy, both before and after treatment with MMSE score evaluation method. Results: After 3 weeks of treatment, B group in the total efficiency was $81.25 \%$, while $\mathrm{C}$ group was $93.75 \%$, which is significantly higher than in A group of $56.25 \%$ and the differences was statistical significance. The three groups before treatment and after treatment with MMSE score changes were obviously difference $(p<0.05)$, where $\mathrm{C}$ group was the most significant changes in MMSE scores $(p<0.05)$. Conclusion: The combined application of oxiracetam and hyperbaric oxygen therapy can improve patient with vascular dementia intellectual impairment and increase the ability of activities in daily living. This method has definite curative effect, high safety as well as can promote clinical promotion.
\end{abstract}

\author{
KEYWORDS \\ Oxiracetam \\ Hyperbaric oxygen \\ Vascular dementia
}

\section{Introduction}

Vascular dementia is a severe cognitive dysfunction syndrome caused by cerebral vascular disease such as ischemic stroke, hemorrhagic stroke, memory, cognition and behavior. It is one of the important causes of senile dementia. The main types of vascular dementia include acute vascular dementia (including multiple infarction dementia, a critical site of infarction dementia, watershed infarction dementia and hemorrhagic dementia), and subacute or chronic vascular dementia (including subcortical atherosclerotic encephalopathy and the autosomal dominant cerebral artery disease associated with subcortical infarction and white matter encephalopathy). The main clinical man-

\section{Copyright (C) 2015 Guiwen Bu}

doi: $10.18686 /$ aem.v4i1.1

Received: December 19, 2014; Accepted: January 8, 2015; Published online: February 13, 2015

This is an open-access article distributed under the terms of the Creative Commons Attribution Unported License (http://creativecommons.org/ licenses/by-nc/4.0/), which permits unrestricted use, distribution, and reproduction in any medium, provided the original work is properly cited.

${ }^{\star}$ Corresponding author: Department of Internal Medicine, The Hospital of Liuyang County, Changsha, Hunan 410000, China. E-mail: guiwen1967@163.com ifestations of patients were cognitive impairment, memory loss, or accompanied by language problem, behavioral changes, limb function and other retardation.

As China's people gradually entered the aging stage, the incidence of cerebrovascular disease is increasing. According to statistics, the number of vascular dementia caused by cerebrovascular disease is increasing for about $26 \%$ [1]. The occurrence of vascular dementia in the elderly health is large. This health problem will impact the quality of life and caused serious influence. As a result, it will bring painful mental burden and huge economic burden to the patient's family and society. Therefore, the combined application of oxiracetam and hyperbaric oxygen on vascular dementia patient on treatment by obtaining the ideal curative effect were observed. The results were reported as follows.

\section{Materials and methods 2.1. General information}

From June 2013 to June 2014, there were 96 cases of vascular dementia patients have been treated. Two criteria have been considered which are inclusion and exclusion. Inclusion criteria are the people who are at age of 40 years old, where sex is not limited. The prevalence of this disease is 3 
months or more. Diagnostic criteria has been developed in order to meet the fourth edition of the American mental disorder diagnosis and Statistical Manual (DSM-IV) and nerve disease and stroke research institute [2]. Exclusion criteria: (1) The evaluation of other diseases which may affect the results; (2) Patients who have complicated severe nerve defects, including aphasia and agnosia problems; (3) Patients who know the cause of dementia from other diseases; (4) Patients with severe heart, liver, kidney and hematopoietic function problems; (5) Patients who are suffering from mental illness and severe diabetes; (6) Patients who has active epilepsy. The baseline examination were taken 2 weeks before by examining the brain cell activator, such as choline piracetam, brain protein hydrolysate, cell Diphosphate Choline and cholinesterase inhibitors and other drugs. Randomly, 96 patients were divided into three groups in which 32 cases in each group of patients who take the hyperbaric oxygen therapy, oxiracetam therapy and combined therapy, respectively. General data of patients in the three groups have no significant difference $(p$ $>0.05$ ), thus there is comparability.

\subsection{Treatment method}

Three groups of patients were given active treatment of basic diseases, including anti platelet to promote cerebral circulation and cerebral protective agent. Hyperbaric oxygen therapy group (A group): 1 hour daily hyperbaric oxygen therapy. Oxiracetam group (B group): Intravenously oxiracetam injection ( $4.0 \mathrm{~g}$ with $250 \mathrm{~mL}$ of physiological saline) one times per day. Hyperbaric oxygen combined with oxiracetam group ( $\mathrm{C}$ group): use the method as mentioned above. Three groups of patients were treated for 3 consecutive weeks. Patients with coronary heart disease and hypertension have been treated with corresponding drugs.

\subsection{Efficacy evaluation criteria}

Before and after the treatment, the two groups of patients undergo Mini Mental State Examination scale (MMSE) assessment with score range 0-30 and for score less than 26 points it is considered having the cognitive barriers. The lower the score, the more serious the degree of dementia. For Activities of Daily Living (ADL) scale, for score less than 16 points is normal, for 17-24 points indicate the problem of brain recognition ability, while for score is equal or greater than 25 , it indicate that the need of brain repairment for loss of function [3].

Results were compared with before and after the treatment; the simple mental state examination scale (MMSE) score increased by 3 points or more is consider excellent while for effective results the MMSE score is increased 1 to 2 points. However, MMSE score is invalid when results are decreased by less than 1 point.

\subsection{Statistical data processing}

SPSS 18.0 software was used to analyze and count data for
Chi-Square test while the measurement data were compared with $t$-test. The single factor analysis of variance among groups was carried out, and the difference was statistically significant $(p<0.05)$.

\section{Results}

\subsection{Clinical curative effect of three groups}

After 3 weeks of treatment, the MMSE score of the two groups were significantly higher than that of the same group before treatment and the difference was statistically significant. The total effective rate of B group was $81.25 \%$, and the total effective rate of $\mathrm{C}$ group was $93.75 \%$, which was significantly higher than that of A group, and also the difference was statistically significant. See Table 1.

Table 1. Clinical efficacy of the three groups [cases (\%)].

\begin{tabular}{lccccc} 
Group & Cases & Excellence & Effective & Invalid & $\begin{array}{c}\text { Total } \\
\text { Efficiency }\end{array}$ \\
\hline A Group & 32 & $10(31.25)$ & $8(25.00)$ & $14(43.75)$ & 56.25 \\
B Group & 32 & $15(46.88)$ & $11(34.38)$ & $6(18.75)$ & $81.25^{*}$ \\
C Group & 32 & $16(50.00)$ & $14(43.75)$ & $2(6.25)$ & $93.75^{* *}$ \\
\hline
\end{tabular}

Note: Compared with A group, ${ }^{*} p<0.05,{ }^{* *} p<0.01$.

\subsection{MMSE scores before and after treatment in three groups}

There were significant differences in MMSE scores between the three groups before and after treatment $(p<0.05)$. Although the change of MMSE scores in $C$ group was significantly higher than that in B group and A group $(p<0.05)$, the different was statistical significance, see Table 2 .

Table 2. MMSE scores of three groups before and after treatment $(\bar{x} \pm s)$.

\begin{tabular}{cccc} 
Group & Cases & $\begin{array}{c}\text { Pre-treatment } \\
\text { score }\end{array}$ & $\begin{array}{c}\text { After treatment } \\
\text { score }\end{array}$ \\
\hline A Group & 32 & $16.86 \pm 3.12$ & $18.13 \pm 3.13^{*}$ \\
B Group & 32 & $16.91 \pm 3.21$ & $18.64 \pm 2.92^{*}$ \\
C Group & 32 & $16.83 \pm 3.13$ & $20.82 \pm 2.81^{* *}$ \\
\hline
\end{tabular}

Note: Compared with the prior treatment, ${ }^{*} p<0.05$; B group and A group, ${ }^{* *} p<0.05$.

\subsection{Side effects}

There were no obvious side effects in the three groups.

\section{Discussion}

Vascular dementia (VD) is one of the mental disorders caused by cerebrovascular disease. The brain function of patients with dementia is decreased for at least a few months, especially with the recession of the intelligence related function, for example, the patient's mood, behavior, memory and cognitive force and other symptoms presented as signs of a recession. The differential diagnostic criteria of vascular dementia are many, including: (1) The cognitive and social function was significantly decreased. 
(2) The basis of cerebrovascular disease was confirmed by medical history, clinical manifestations, and the auxiliary examination. (3) Dementia occurred within 3-6 months after cerebral vascular disease [4].

Differential diagnosis of typical symptoms of Vascular dementia is categorized into four types: (1) Alzheimer's disease $(\mathrm{AD})$ and its insidious onset: slow progress, loss outstanding memorycognitive dysfunction and personality changes. Neuroimaging findings show significant cerebral cortex atrophy with ischemic volume table is less than or equal to 4 points (improved ischemia volume table is less than or equal to 2 points) to support the diagnosis of AD; (2) Pick's disease, ascended to the throne of dementia show early obvious personality change and social behavior disorders, language impairment, memory and cognitive function disorder relatively late. CT or MRI is mainly significant for frontal and / or temporal lobe atrophy; (3) Dementia with Lewy Bodies (DLB) where the volatility of cognitive impairment, repeated vivid visual hallucinations and extrapyramidal symptoms. However, imaging observation show no infarction and no signs of localization neurological examination; (4) Parkinson's disease with dementia and its early appear extrapyramidal involvement symptoms such as tremor, myotonia, in attention, calculation, visual spatial memory, etc. mainly damaged [5].

Oxiracetam in chemical structure belongs to a kind of amino acid derivatives. The cerebral cortex and hippocampus that play protection and activation can activate adenylate cyclase which increases the synthesis of protein and nucleic acid in the brain and acting on aspartate (NMDA) receptor. Thus, it will affect long-term potentiation (LTP) generation by increased acetylcholine in cortex and hippocampus and the transport of choline uptake. Besides, it is also increase affinity, promote brain metabolism and improve cerebral oxygen utilization of glucose in which contribute to the repair of nerve cell function. Moreover, oxiceratam also can improve memory and learning ability of patients with mental retardation by activation, protection and repair of nerve cells so that the brain has a protective effect on hypoxia [6]. Oxiracetam can improve the patient's mental state and reduce the degree of dementia with no side effect.

Hyperbaric oxygen can also be effective in the treatment of vascular dementia. Hyperbaric oxygen therapy is the process of treating the disease with high concentrations of oxygen above than that in the atmospheric pressure. The therapeutic mechanism are: (1) High pressure oxygen can effectively improve blood pressure by enhanced blood oxygen dispersion ability and correct brain damage to the hypoxic state; (2) Cerebral blood flow by improving the blood flow of vertebral artery which can activate the network system and oxygen partial pressure. After all, this method can increase the oxygen partial pressure and oxygen content of the body which relieve the oxygen supply of brain tissue and increase the blood oxygen supply to improve microcirculation abnormality. Thus it exerts the effect of preventing and treating vascular dementia [7].

The results of this study showed that the change of MMSE scores of the combined treatment group was the most significant, and there was statistical significance. In summary, combined oxiracetam with hyperbaric oxygen therapy can be effective in improving vascular dementia patients with mental retardation and increase the ability of activities in daily life. The efficacy and safety of the method is proved in order to be as clinical promotion.

\section{References}

1. Yi Y, Ye D. Less Dan combined with oxiracetam in treatment of vascular dementia clinical observation. Chinese Medical Science. 2012;18(10):77.

2. Lu Y, Wan. Oxiracetam in treatment of vascular dementia 80 example clinical curative effect observation. Journal of Chinese Pharmaceutical Sciences. 2011;13(2):59-62.

3. Liu S, Sun X, Xia C. Clinical treatment and differentiation of vascular dementia. 2010;4(2):33-34.

4. Xing $X$. The cause and mechanism of vascular dementia. Journal of Shenyang Medical College. 2012;21(2):117118.

5. Shi LS, Chen C. Oxiracetam capsules treatment mild vascular dementia efficacy and safety. China Practical Medicine. 2009;4(24):57-58.

6. Xiao X. Laci Staw and the effect on vascular dementia. Chinese Contemporary Medicine. 2012;23(17):64.

7. Wei $\mathrm{H}$, Wang $\mathrm{S}$. Research progress on the mechanism of action of kidney drugs on vascular dementia. Journal of Guangxi Traditional Chinese Medical University. 2012;25(2):73-75. 